\title{
DYNAMIC MODELLING OF A VIBRATORY ASPHALT COMPACTOR AND ESTIMATION OF CONTACT FORCES WRENCH
}

\author{
Charles-Eric Lemaire ${ }^{*}$ Pierre-Olivier Vandanjon * \\ Maxime Gautier** \\ * Laboratoire Central des Ponts des Chaussées, BP 4129, \\ 44341 Bouguenais Cedex, France \\ ** Institut de Recherche en Communication et Cybernétique \\ de Nantes, BP 92101, 44321 Nantes Cedex 3, France
}

\begin{abstract}
This paper deals with the dynamic modelling of a vibratory asphalt compactor. A compactor is a machine of road building site. Its task is to increase the density of the asphalt. The aim of the study is to measure contact forces wrenches between the drums of the compactor and the bituminous mix. These drums vibrate. Traditional techniques of robotics are used to model the compactor as an articulated mechanical system. In order to reach our objective, the dynamic model is limited to the clamp-drum unit. Moreover, this model is adapted to our system of measurements by replacing some lagrangian variables by some Euler variables. In this manner, it is proved that it is possible to get the contact forces wrench. During a real worksite, it has been possible for the first time to measure the contact forces applied by a compactor. Copyright ${ }^{\circledR 2} 2005$ IFAC.
\end{abstract}

Keywords: Dynamic modelling, mobile robots, contact force

\section{INTRODUCTION}

In road construction, material is spread then compacted. Compacting is a critical task of this process, guarantees lifetime of the road. To achieve this task, a worksite machine is used : a vibratory asphalt compactor (Fig.1). Compaction is achieved by the operating weight and the vibratory system of the machine. Now, a reliable, realtime system to control asphalt compaction does not exist when they are top courses of the road. A modelling of a compactor as an articulated mechanical system without vibration (Guillo et al., 1999) enables us to develop a low-cost system (Lemaire et al., 2003) to measure the rolling resistance (Delclos et al., 2001). In this paper, a further modelling witch take into account vibration is presented. With this model, it is possible to measure contact forces wrenches applied by the compactor to asphalt in 2D. To carry out this measure, a complete model of the compactor is not necessary, a drum model is enough, with the hypothesis of plan motion of the compactor which is adequate to considered applications. The dynamic model is based on a geometric modelling. The first part of this article deals with geometric modelling of the compactor. The second part describes the traditional calculation of dynamic model. In the third part, this modelling is adapted to the case of the compactor in the objective to know contact forces wrench.

\section{GEOMETRIC DESCRIPTION OF THE COMPACTOR}

In this part, the geometric model of a typical compactor -Caterpillar CB544 (Fig. 1)- is described. 


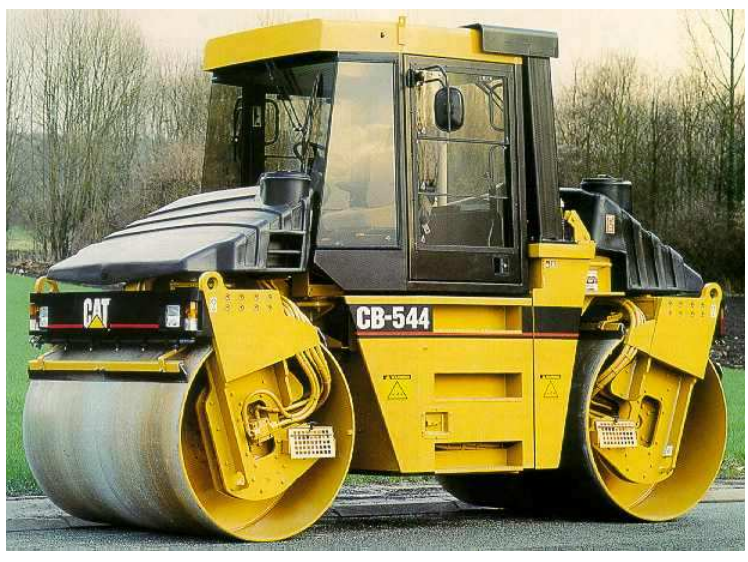

Fig. 1. A typical compactor: Caterpillar CB544

We consider in the elements that link the chassis - or main body - to the ground. The compactor is then composed of:

- the chassis

- the steering system

- the two drums, each drum is composed of two half-drum and a circular exciter system

This description makes use of the most influent degrees of freedom characterizing the compactor dynamics. These degrees of freedom are modeled according to the modified Denavit and Hartenberg (MDH) notations (Khalil and Dombre, 2002), it leads to the geometric model. The second part connects this complex model to the principal degrees of freedom of the compactor.

\section{1 geometric model}

The compactor is considered as a multi-body tree structure, with $n$ bodies, where the halfdrums represent the terminal links. Each body $B_{j}$ is linked to its antecedent with a joint which represents an elementary degree of freedom either a translational or a revolute, the joint can be rigid or elastic. A body (or a link) can be real or virtual, the virtual bodies are introduced to describe complex joints or projection frames. We define a reference frame $R_{j}$ (with origin $O_{j}$ and main axes $x_{j}, y_{j}, z_{j}$ ) attached to each body $B_{j}$. The $z_{j}$ axis is defined along the axis of joint $j$. The axis $u_{k}$ is defined along the common normal between $z_{j}$ and $z_{k}$, where link $j$ is the antecedent of link $k$, denoted by $j=a(k)$. The $x_{j}$ axis is defined arbitrarily along one of the axes $u_{k}$, with $a(k)=j$. The $(4 \times 4)$ homogenous transformation matrix ${ }^{i} T_{j}$ between two consecutive frames $R_{i}$ and $R_{j}$, with $i=a(j)$ is defined with the following six parameters (Khalil and Dombre, 2002) (Fig.2):

- $\gamma_{j}$ : angle between $x_{i}$ and $u_{j}$ around the axis $z_{i}$

- $b_{j}$ : distance between $x_{i}$ and $u_{j}$ along $z_{i}$,

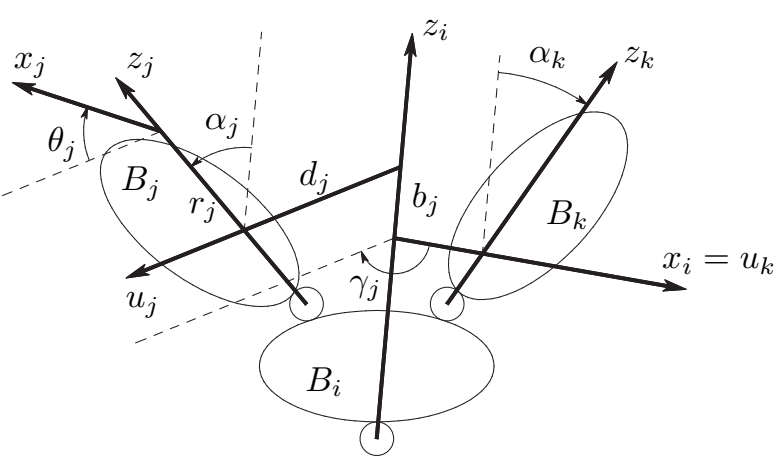

Fig. 2. The geometric parameters

- $\alpha_{j}$ : angle between $z_{i}$ and $z_{j}$ around the axis $u_{j}$

- $d_{j}$ : distance from $z_{i}$ to $z_{j}$ along $u_{j}$,

- $\theta_{j}$ : angle between $u_{j}$ and $x_{j}$ around the axis $z_{j}$

- $r_{j}$ : distance from $u_{j}$ to $x_{j}$ along $z_{j}$.

When $x_{i}$ is taken along $u_{j}$, the parameters $\gamma_{j}$ and $b_{j}$ are equal to zero. This is always the case for all the frames of serial robots. The homogenous $(4 \times 4)$ transformation matrix ${ }^{i} T_{j}$ between frames $i$ and $j$ is given as:

$$
{ }^{i} T_{j}=\left[\begin{array}{cc}
{ }^{i} A_{j} & { }^{i} P_{j} \\
0 & 0
\end{array}\right]
$$

with

${ }^{i} A_{j}=\left[\begin{array}{ccc}C \gamma_{j} C \theta_{j}-S \gamma_{j} C \alpha_{j} S \theta_{j} & -C \gamma_{j} S \theta_{j}-S \gamma_{j} C \alpha_{j} C \theta_{j} & S \gamma_{j} S \alpha_{j} \\ S \gamma_{j} C \theta_{j}-C \gamma_{j} C \alpha_{j} S \theta_{j} & -S \gamma_{j} S \theta_{j}+C \gamma_{j} C \alpha_{j} C \theta_{j} & -C \gamma_{j} S \alpha_{j} \\ S \alpha_{j} S \theta_{j} & S \alpha_{j} C \theta_{j} & C \alpha_{j}\end{array}\right]$

and

$$
{ }^{i} P_{j}=\left[\begin{array}{c}
d_{j} C \gamma_{j}+r_{j} S \gamma_{j} S \alpha_{j} \\
d_{j} S \gamma_{j}-r_{j} C \gamma_{j} S \alpha_{j} \\
r_{j} C \alpha_{j}+b_{j}
\end{array}\right]
$$

Where:

- ${ }^{i} A_{j}$ is the $(3 \times 3)$ orientation matrix of frame $j$ with respect to frame $i$,

- ${ }^{i} P_{j}$ is the $(3 \times 1)$ vector defining the origin of frame $j$ with respect to frame $i$

- $C x=\cos (x)$ and $S x=\sin (x)$

The generalized coordinate of joint $j$ is denoted $q_{j}$, it is equal to $r_{j}$ if $j$ is translational and $\theta_{j}$ if $j$ is revolute. It can be written by the following relation: $q_{j}=\sigma_{j} r_{j}+\bar{\sigma}_{j} \theta_{j}$ where $\sigma_{j}=1$ if joint $j$ is translational, and $\sigma_{j}=0$ if joint $j$ is revolute, $\bar{\sigma}_{j}=1-\sigma_{j}$. If there is no degree of freedom between two frames; fixed with respect to each other, we take $\sigma_{j}=2$, it means that the time derivative of $q_{j}$ is zero.

According to this formalism, the geometric description of the compactor is given by Fig.3. The parameters of the geometric description are given by Table 1 . The explanation of this modelling is the object of the following paragraphs. 


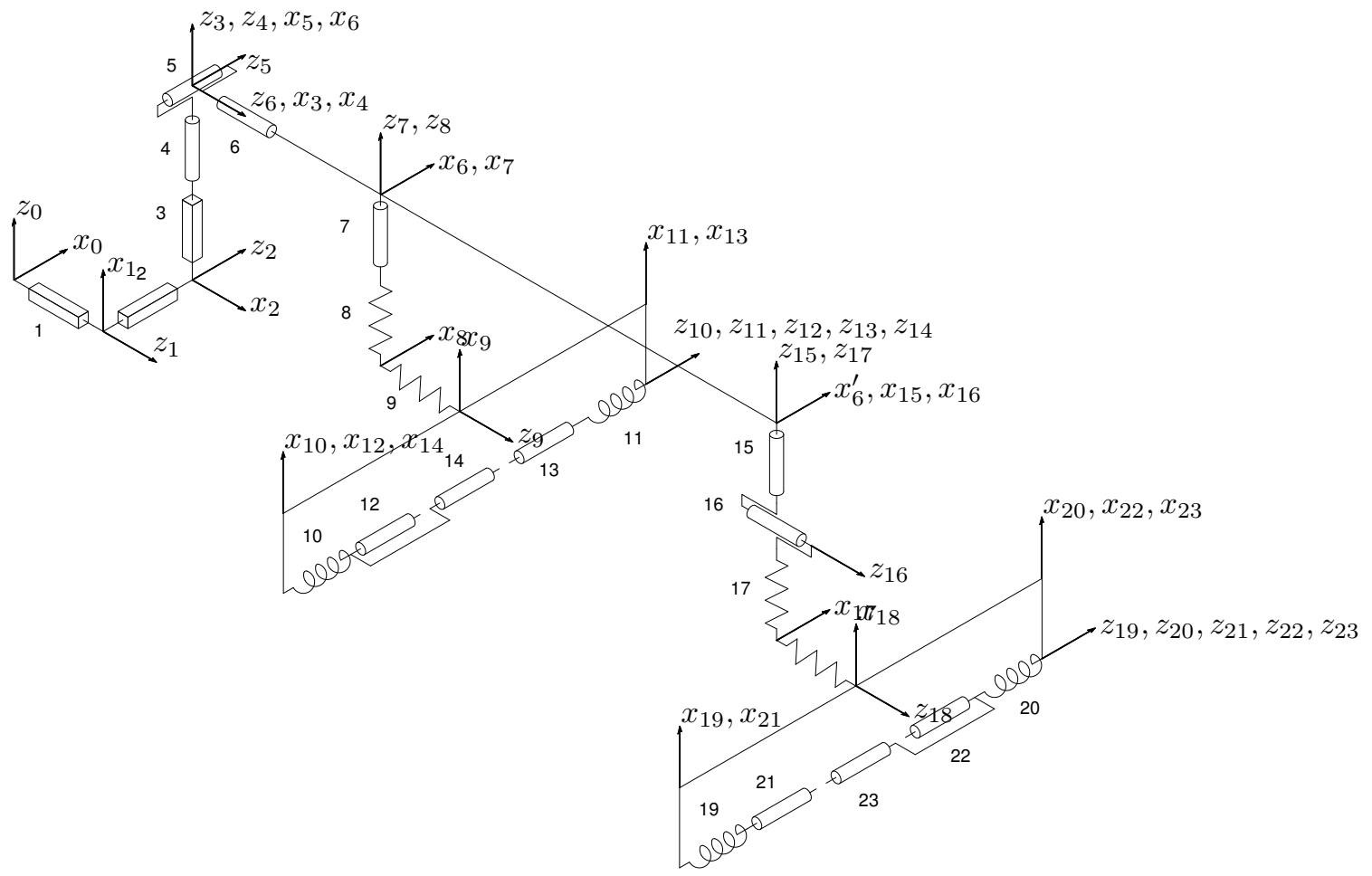

Fig. 3. Tree structure of the compactor

Table 1. Geometric parameters of the compactor

\begin{tabular}{cccccccccc}
$\mathrm{j}$ & $\mu_{j}$ & $\sigma_{j}$ & $a_{j}$ & $\gamma_{j}$ & $b_{j}$ & $\alpha_{j}$ & $d_{j}$ & $\theta_{j}$ & $r_{j}$ \\
\hline 1 & 0 & 1 & 0 & 0 & 0 & $\frac{\pi}{2}$ & 0 & $\frac{\pi}{2}$ & $q_{1}$ \\
2 & 0 & 1 & 1 & 0 & 0 & $\frac{\pi}{2}$ & 0 & $\frac{\pi}{2}$ & $q_{2}$ \\
3 & 0 & 1 & 2 & 0 & 0 & $\frac{\pi}{2}$ & 0 & 0 & $q_{3}$ \\
4 & 0 & 0 & 3 & 0 & 0 & 0 & 0 & $q_{4}$ & 0 \\
5 & 0 & 0 & 4 & 0 & 0 & $-\frac{\pi}{2}$ & 0 & $q_{5}$ & 0 \\
6 & 0 & 0 & 5 & 0 & 0 & $-\frac{\pi}{2}$ & 0 & $q_{6}$ & 0 \\
7 & 1 & 0 & 6 & 0 & 0 & $-\frac{\pi}{2}$ & 0 & $q_{7}$ & 0 \\
8 & 0 & 1 & 7 & 0 & 0 & 0 & 0 & 0 & $q_{8}$ \\
9 & 0 & 1 & 8 & 0 & 0 & $\frac{\pi}{2}$ & 0 & $\frac{\pi}{2}$ & $q_{9}$ \\
10 & 0 & 0 & 9 & 0 & 0 & $\frac{\pi}{2}$ & 0 & $q_{10}$ & $-\frac{L}{2}$ \\
11 & 0 & 0 & 9 & 0 & 0 & $\frac{\pi}{2}$ & 0 & $q_{11}$ & $\frac{L}{2}$ \\
12 & 1 & 0 & 10 & 0 & 0 & 0 & 0 & $q_{12}$ & 0 \\
13 & 1 & 0 & 11 & 0 & 0 & 0 & 0 & $q_{13}$ & 0 \\
14 & 1 & 0 & 10 & 0 & 0 & 0 & 0 & $q_{14}$ & 0 \\
15 & 1 & 0 & 6 & 0 & $\mathrm{D}$ & $-\frac{\pi}{2}$ & 0 & $q_{15}$ & 0 \\
16 & 0 & 0 & 15 & 0 & 0 & $\frac{\pi}{2}$ & 0 & $q_{16}$ & 0 \\
17 & 0 & 1 & 16 & 0 & 0 & $-\frac{\pi}{2}$ & 0 & 0 & $q_{17}$ \\
18 & 0 & 1 & 17 & 0 & 0 & $\frac{\pi}{2}$ & 0 & $\frac{\pi}{2}$ & $q_{18}$ \\
19 & 0 & 0 & 18 & 0 & 0 & $\frac{\pi}{2}$ & 0 & $q_{19}$ & $-\frac{L}{2}$ \\
20 & 0 & 0 & 18 & 0 & 0 & $\frac{\pi}{2}$ & 0 & $q_{20}$ & $\frac{L}{2}$ \\
21 & 1 & 0 & 19 & 0 & 0 & 0 & 0 & $q_{21}$ & 0 \\
22 & 1 & 0 & 20 & 0 & 0 & 0 & 0 & $q_{22}$ & 0 \\
23 & 1 & 0 & 20 & 0 & 0 & 0 & 0 & $q_{23}$ & 0 \\
\hline & & & & & & & & &
\end{tabular}

2.2 study of the degrees of freedom of the compactor

In this paragraph, the figure 3 is explained according to the main degrees of freedom of the compactor.

Motions of the drums with respect to the chassis

- each half-drum turns around its axis (joints 12,13 and 21,22),
- the circular exciter system turns around its axis (joints 14 and 23),

- each drum (made up of the two half-drum and one circular exciter system) is suspended by silent blocs - with four degrees of freedom to a clamp (joints 8,9,10,11 and 17,18,19,20),

- the back clamp is articulated with respect to the frame by a revolute joint(articulation 7),

- the front clamp is articulated with respect to the frame by two revolute joints, to the steering motion is added a pendular motion to compensate for the warping of the ground (joints 15 and 16).

Motions of the chassis with respect to ground The compactor motion with respect to ground is described by three translations and three rotations degrees of freedom which are called (Figure $3)$ :

- longitudinal translation (joint 1),

- lateral translation (joint 2),

- vertical translation (joint 3),

- yaw, rotation around the vertical axis (joint 4),

- pitch, rotation around the transversal axis (joint 5),

- roll, rotation around the longitudinal axis (joint 6),

\section{DYNAMIC MODELLING}

From the geometric model, we can ccompute a dynamic model. We describe this step because we 
present in the following section, which adaptation was carried to the method to fulfill our objective: to measure the contact forces wrench.

The description of dynamic modelling needs the presentation of the parameters associated with each body. In the continuation, we will need to distinguish two types of formalisms: Lagrangian formalism and Newton-Euler formalism. The dynamic model is then described. The effective calculation of this dynamic model is based on the equations of Newton-Euler. It is the object of the last paragraph of this part.

\subsection{Dynamic parameters}

A set of ten inertial parameters is associated with each real body $B_{j}$, it consists of:

- the mass $M_{j}$

- the 6 components of the inertia matrix $J_{j}$ given in Frame $R_{j}$, they are denoted by $X X_{j}$ $X Y_{j} X Z_{j} Y Y_{j} Y Z_{j} Z Z_{j}$

- the first moments parameters $M X_{j} M Y_{j}$ $M Z_{j}$ with respect to frame $R_{j}$

When there is an elastic joint between $R_{i}$ and $R_{j}$ it is necessary to define some elastic parameters:

- the stiffness $k_{j}$ of the joint

- the damping coefficient $h_{j}$

- the Coulomb coefficient $f s_{j}$

The vector of standard dynamic parameters $\mathrm{Xs}_{\mathrm{s}}$ is composed of the previous parameters of all the links.

\subsection{Formalisms}

In general, the dynamic model is expressed with the Lagrange formalism and/or the Newton-Euler formalism. The Lagrange formalism expresses the movement of each body in terms of the joint generalized coordinates $q=\left[q_{1} \cdots q_{n}\right]^{T}$, its first and second derivatives, the external wrenches applied on the system $F_{e}$ and the vector of dynamic parameters $X_{S}$. It is given by:

$$
f\left(q, \dot{q}, \ddot{q}, F_{e}, X_{S}\right)=0
$$

The Euler formalism expresses the movement of a body with its rotational speed, rotational acceleration, translational acceleration and the current position $[\omega, \dot{\omega}, \dot{V}, \Phi]$. It can be written as:

$$
f\left(\omega, \dot{\omega}, \dot{V}, \Phi, F_{e}, X_{S}\right)=0
$$

\subsection{The Lagrange dynamic model}

The inverse dynamic model is obtained with the following general equation:

$$
\Gamma+Q=\Gamma^{e}+\Gamma^{f}+A(q) \ddot{q}+C(q, \dot{q})
$$

Where:

- $\Gamma$ is the joint forces or torques vector,

- $Q$ is the vector of generalized efforts representing the projection of the external wrenches on the joint axes, it is calculated with:

$$
Q=-\Sigma J_{j}^{T}(q) F_{e j}
$$

- $J_{j}(q)$ is the Jacobian matrix of frame $R_{j}$,

- $F_{e j}$ is the external wrench (forces $f_{e j}$ and moments $m_{e j}$ ) applied by body $B_{j}$ on the environment,

- $\Gamma^{f}$ is the friction force,

- $\Gamma^{e}$ is the joint elastic force,

- $A(q)$ is the inertia matrix of the system,

- $C(q, \dot{q})$ is the vector of Coriolis, centrifugal and gravity forces.

The $j^{\text {th }}$ element of $\Gamma^{e}$, is written as:

- $\Gamma_{j}^{e}=k_{j} q_{j}$ if $j$ is an elastic joint, with $q_{j}$ the joint coordinate with respect to the original position and $k_{j}$ the stiffness of joint $j$,

- $\Gamma_{j}^{e}=0$ if $j$ is not an elastic joint.

Frictions are modeled by a viscous coefficient $h_{j}$ and a Coulomb coefficient $f s_{j}$ :

$$
\Gamma_{j}^{f}=h_{j} \dot{q}_{j}+f s_{j} \operatorname{sign}\left(\dot{q}_{j}\right)
$$

\subsection{Practical calculation of the Lagrange dynamic model}

The Lagrange model is calculated classically with the Lagrange equation after calculating the kinetic and potential energy of all the elements of the mechanical system, and by calculating the generalized forces using equation (5) or by the use of the virtual work principle (Guillo et al., 1999). It can be calculated more efficiently using a recursive algorithm based on the Newton-Euler equation, after expressing the link velocities and accelerations in terms of joint positions, velocities and accelerations (Khalil and Kleinfinger, 1987). This algorithm consists of two recursive calculations. The forward one calculates the total forces and moments on each body, while the backward one leads to calculate the joint torques. The forward recursive calculation is summarized as follows: for $j=1, \cdots, n$, we calculate successively:

$$
\begin{gathered}
{ }^{j} \omega_{i}={ }^{j} A_{i}{ }^{i} \omega_{i} \\
{ }^{j} \omega_{j}={ }^{j} \omega_{i}+\bar{\sigma}_{j} \dot{q}_{j}{ }^{j} a_{j} \\
{ }^{j} \dot{\omega}_{j}={ }^{j} A_{i}{ }^{i} \dot{\omega}_{i}+\bar{\sigma}_{j}\left(\ddot{q}_{j}{ }^{j} a_{j}+{ }^{j} \omega_{i} \times \dot{q}_{j}{ }^{j} a_{j}\right) \\
{ }^{j} \dot{V}_{j}={ }^{j} A_{i}\left({ }^{i} \dot{V}_{i}+\left({ }^{i} \tilde{\dot{\omega}}_{i}+{ }^{i} \tilde{\omega}_{i}{ }^{i} \tilde{\omega}_{i}\right)^{i} P_{j}\right) \\
+\sigma{ }_{j}\left(\ddot{q}_{j}{ }^{j} a_{j}+2^{j} \omega_{i} \times \dot{q}_{j}{ }^{j} a_{j}\right) \\
{ }^{j} F_{j}=\mathcal{M}_{j}{ }^{j} \dot{V}_{j}+\left({ }^{j} \tilde{\dot{\omega}}_{j}+{ }^{j} \tilde{\omega}_{j}{ }^{j} \tilde{\omega}_{j}\right)^{j} M S_{j}
\end{gathered}
$$




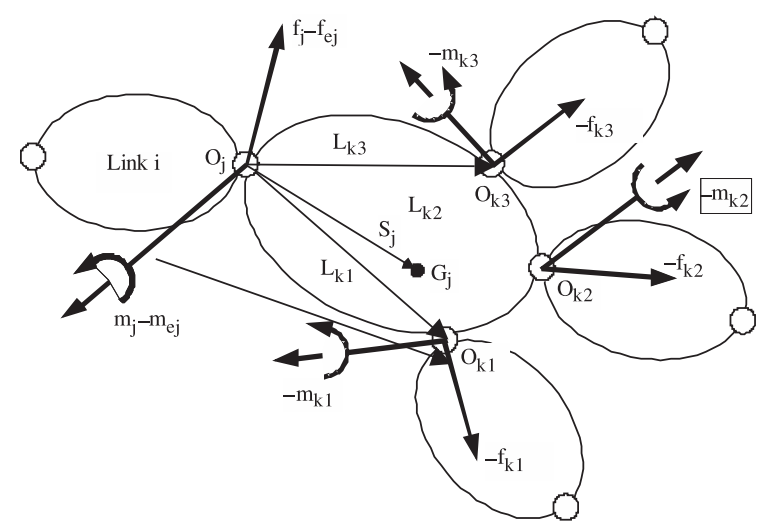

Fig. 4. Forces and moments acting on a link of a tree structure

$$
{ }^{j} M_{j}={ }^{j} J_{j}{ }^{j} \dot{\omega}_{j}+{ }^{j} \omega_{j} \times{ }^{j} J_{j}{ }^{j} \omega_{j}+{ }^{j} M S_{j} \times{ }^{j} \dot{V}_{j}
$$

With the upper left exponent denotes the projection frame, and:

- $i=a(j)$,

- $\dot{\omega}_{j}$ the angular acceleration of body $j$,

- $\omega_{j}$ the angular velocity of body $j$,

- $\dot{V}_{j}$ the acceleration of $O_{j}$, origin of frame $j$,

- $F_{j}$ total forces applied on body $j$

- $M_{j}$ total moments applied on body $j$ with respect to $O_{j}$,

- ${ }^{j} A_{i}$ the $(3 \times 3)$ orientation matrix of frame $R_{i}$ in $R_{j}$

- $a_{j}$ is the unit vector along $z_{j}$, thus ${ }^{j} a_{j}=$ $\left[\begin{array}{lll}0 & 0 & 1\end{array}\right]^{T}$,

- $\mathcal{M}_{j}$ is the mass of body $j$,

- $J_{j}$ is the inertia matrix of body $j$, given in frame $R_{j}$,

- $M S_{j}$ the vector of first moments of inertia of $B_{j}$ around $O_{j}$

- $\tilde{S}$ is the skew-symmetric matrix defined from the components of the $(3 \times 1)$ vector $S$ by:

$$
\tilde{S}=\left[\begin{array}{ccc}
0 & -s_{z} & s_{y} \\
s_{z} & 0 & -s_{x} \\
-s_{y} & s_{x} & 0
\end{array}\right]
$$

The forward calculation is initialized with ${ }^{0} \omega_{0}=$ $0,{ }^{0} \dot{\omega}_{0}=0$, whereas the translational acceleration of frame 0 will be set equal to gravity $g$ with opposite sign, in order to take into account automatically the effect of the gravity forces, thus ${ }^{0} \dot{V}_{0}=-g$.

The backward recursive equations, for $j=$ $n, \cdots, 1$ calculates the forces ${ }^{j} f_{j}$ and moments ${ }^{j} m_{j}$ exerted on body $B_{j}$ by its antecedent body $B_{i}$ (Fig. 4), it gives:

$$
\begin{gathered}
{ }^{j} f_{j}={ }^{j} F_{j}+{ }^{j} f_{e j}+\sum_{s(j)}{ }^{j} f_{s(j)} \\
{ }^{i} f_{j}={ }^{i} A_{j}{ }^{j} f_{j}
\end{gathered}
$$

$$
\begin{aligned}
& { }^{j} m_{j}={ }^{j} M_{j}+{ }^{j} m_{e j} \\
& +\sum_{s(j)}\left({ }^{j} A_{s(j)}{ }^{s(j)} m_{s(j)}+{ }^{j} \tilde{P}_{s(j)}{ }^{j} f_{s(j)}\right)
\end{aligned}
$$

The joint forces (or torques) are obtained by projecting ${ }^{i} f_{j}\left(\right.$ or ${ }^{i} m_{j}$ ) on the joint axis $z_{j}$ and by taking into account the friction and elasticity effects as follows:

$$
\Gamma_{j}=\left(\sigma_{j}^{j} f_{j}+\tilde{\sigma}_{j}^{j} m_{j}\right)^{T j} a_{j}+\Gamma_{j}^{f}+\Gamma_{j}^{e}
$$

With:

- $s(j)$ indicates the body whose antecedent is body $B_{j}$,

- $f_{e j}$ the external force applied by body $B_{j}$ on the environment,

- $m_{e j}$ the external moments applied by body $B_{j}$ on the environment,

- $f_{j}$ the forces applied by body $B_{j}$ on body $B_{i}$, with $a(j)=i$,

- $m_{j}$ the torques applied by body $B_{j}$ on body $B_{i}$.

This backward calculation is initialized by putting ${ }^{j} f_{j},{ }^{j} m_{j}$ equal to zero for the terminal links. We note that the contact forces between the drum and the ground will be taken into account through $f_{e j}$ and $m_{e j}$ of the terminal links (the half-drums). The projection of these forces on the joint axes will be obtained systematically without application of equation (5) as would be the case if the Lagrange equation is used.

It has to be noted that this algorithm can be programmed numerically or symbolically. To optimize its number of operations, we use customized symbolic techniques to implement it (Khalil and Kleinfinger, 1987). It can be proved that the dynamic model is linear with respect to the vector of standard dynamic parameters $X_{S}$, thus equation (4) can be rewritten as:

$$
L=\Gamma+Q=D_{S} X_{S}
$$

Where the matrix $D_{S}$ is a function of $(q, \dot{q}, \ddot{q})$.

\section{APPLICATION TO THE COMPACTOR}

The example presented here is that of the front drum of the compactor. It is considered twodimensional motion of the compactor. This assumption is realistic taking into account the operation of the compactors on building site.

Our objective is to know contact forces wrenches between the drums and the ground. A solution is to calculate these efforts thanks to the dynamic model of the compactor. According to the equation (18) if:

- $X_{S}$ is identified, 
- $D_{S}$ is computed from $(q, \dot{q}, \ddot{q})$ which have to be measured or estimated,

- $\Gamma$ is measured.

Then we can computed $Q$ the projection of contact forces on joint axis.

We will show that it is not necessary to know the complete model to calculate contact forces wrenches. For that we will study the equations of the dynamic model utilizing the drum-clamp unit. The expression of models will be simplified by combining the measurement of Euler variables and Lagrangian variables.

To calculate the equations of the dynamic model, the equations of Newton-Euler (17) are used between bodies 17 to 23 . The recurrence is initialized with the Euler variables of the body 16: $\omega_{16}$, $\dot{\omega}_{16}, \dot{V}_{16}$. It is thus possible to calculate joint torques $\Gamma_{17}$ to $\Gamma_{23}$, an analysis of these equations proves that the expressions of $\Gamma_{17}, \Gamma_{18}, \Gamma_{21}$ and $\Gamma_{22}$ (equations 19 to 22 ) are enough to compute contact forces between the front drum and the ground.

Taking into account the instrumentation, it was easier to measure rather the Euler variables of the engine supports (body 19 and 20) than that of the drum clamp (body 16). In order to replace the terms intervening in the equations by the data measured, the variables $\omega_{16}, \dot{\omega}_{16}, \dot{V}_{16}$ are expressed with respect to $\omega_{19}, \dot{\omega}_{19}, \omega_{20}, \dot{\omega}_{20}, \dot{V}_{20}$.

$\Gamma_{17}=\left(\dot{V}_{20}^{x} \cos \left(q_{20}\right)-\dot{V}_{20}^{y} \sin \left(q_{20}\right)\right) M R_{19}+h_{17} \dot{q}_{17}+k_{17} q_{17}$

$-\left(\left(\omega_{20}^{z}+\dot{q}_{23}\right)^{2} \cos \left(q_{20}+q_{23}\right)+\left(\dot{\omega}_{20}^{z}+\ddot{q}_{23}\right) \sin \left(q_{20}+q_{23}\right)\right) M X_{23}$

$+\left(\left(\omega_{20}^{z}+\dot{q}_{23}\right)^{2} \sin \left(q_{20}+q_{23}\right)-\left(\dot{\omega}_{20}^{z}+\ddot{q}_{23}\right) \cos \left(q_{20}+q_{23}\right)\right) M Y_{23}$

$+F X_{21}+F X_{22}$

$\Gamma_{18}=\left(\dot{V}_{20}^{x} \sin \left(q_{20}\right)+\dot{V}_{20}^{y} \cos \left(q_{20}\right)\right) M R_{19}+h_{18} \dot{q}_{18}+k_{18} q_{18}$

$-\left(\left(\omega_{20}^{z}+\dot{q}_{23}\right)^{2} \sin \left(q_{20}+q_{23}\right)-\left(\dot{\omega}_{20}^{z}+\ddot{q}_{23}\right) \cos \left(q_{20}+q_{23}\right)\right) M X_{23}$

$-\left(\left(\omega_{20}^{z}+\dot{q}_{23}\right)^{2} \cos \left(q_{20}+q_{23}\right)+\left(\dot{\omega}_{20}^{z}+\ddot{q}_{23}\right) \sin \left(q_{20}+q_{23}\right)\right) M Y_{23}$

$+F Y_{21}+F Y_{22}$

$\Gamma_{21}=\left(\dot{\omega}_{19}^{z}+\ddot{q}_{21}\right) Z Z_{21}+h_{21} \dot{q}_{21}+f s_{21} \operatorname{sign}\left(\dot{q}_{21}\right)+C Z_{21}$

$\Gamma_{22}=\left(\dot{\omega}_{20}^{z}+\ddot{q}_{22}\right) Z Z_{22}+h_{22} \dot{q}_{22}+f s_{22} \operatorname{sign}\left(\dot{q}_{22}\right)+C Z_{22}$

with :

$$
M R_{19}=M_{19}+M_{20}+M_{21}+M_{22}+M_{23}
$$

The mechanical structure of the compactor does not make it possible to differentiate the contribution from contact forces between each half-drum. So, it is the sums of the resultants $\left(F X_{21}+F X_{22}\right.$ and $F Y_{21}+F Y_{22}$ ) which are computed (equations 19 and 20). Considering the equations (21) and (22) it is possible to compute the couples $C Z_{21}$ and $C Z_{22}$.

\section{CONCLUSION}

In this paper, the dynamic modelling of a vibratory compactor under the articulated mechanical system formalism is described. This modelling is adapted to the measurement of contact forces wrenches. To achieve this goal,

- the model is restricted with the clamp-drum unit,

- Lagrangian variables necessary to the calculation of the model are replaced by Euler variable easier to measure.

Thank to this modelling, we can compute the torque of the contact force wrench in $2 \mathrm{D}$ on the condition of identifying the parameters of the model. This step of identification through dedicated experiments has been done. Thanks to this modelling study, we could measure on a road building site the torque of the efforts of contact.

\section{ACKNOWLEDGEMENTS}

The authors would like to thanks Michel Froumentin from the Centre d'expérimentation Routière (Rouen, France), Ario Kordestani and Laurent Rocher from the Caterpillar company for fruitful discussions concerning compactor modelling

\section{REFERENCES}

Delclos, A., P-O. Vandanjon, F. Peyret and M. Gautier (2001). Estimating the degree of compaction of asphalt using proprioceptive sensor and dynamic model. In: Proceedings of International Symposium on Automation in Road Construction. Krakow, Poland.

Guillo, E., M. Gautier and F. Boyer (1999). Dynamic modelling and simulation of a compactor. In: Proceedings of 14th IFAC World Congress. Beijing, China. pp. 281-286.

Khalil, W. and E. Dombre (2002). Modeling, identification and control of robots. Hermès Penton. London-U.K.

Khalil, W and J.-F Kleinfinger (1987). Minimum operations and minimum parameters of the dynamic model of tree structure robots. IEEE Journal of Robotics and Automation pp. 517526.

Lemaire, C-E., P-O. Vandanjon and M. Gautier (2003). Dynamic identification of a compactor using splines data processing. In: Proceedings of System Identification. Rotterdam, Nederlands. 